A single active nanoelectromechanical tuning fork front-end radio-frequency receiver

This article has been downloaded from IOPscience. Please scroll down to see the full text article.

2012 Nanotechnology 23225501

(http://iopscience.iop.org/0957-4484/23/22/225501)

View the table of contents for this issue, or go to the journal homepage for more

Download details:

IP Address: 128.178.19.29

The article was downloaded on 23/05/2013 at 15:00

Please note that terms and conditions apply. 


\title{
A single active nanoelectromechanical tuning fork front-end radio-frequency receiver
}

\author{
Sebastian T Bartsch, A Rusu and Adrian M Ionescu \\ Nanoelectronic Devices Laboratory, Ecole Polytechnique Fédéral de Lausanne (EPFL), 1015 Lausanne, \\ Switzerland \\ E-mail: adrian.ionescu@epfl.ch
}

Received 26 February 2012, in final form 18 April 2012

Published 10 May 2012

Online at stacks.iop.org/Nano/23/225501

\begin{abstract}
Nanoelectromechanical systems (NEMS) offer the potential to revolutionize fundamental methods employed for signal processing in today's telecommunication systems, owing to their spectral purity and the prospect of integration with existing technology. In this work we present a novel, front-end receiver topology based on a single device silicon nanoelectromechanical mixer-filter. The operation is demonstrated by using the signal amplification in a field effect transistor (FET) merged into a tuning fork resonator. The combination of both a transistor and a mechanical element into a hybrid unit enables on-chip functionality and performance previously unachievable in silicon. Signal mixing, filtering and demodulation are experimentally demonstrated at very high frequencies $(>100 \mathrm{MHz})$, maintaining a high quality factor of $Q=800$ and stable operation at near ambient pressure $(0.1 \mathrm{~atm})$ and room temperature $(T=300 \mathrm{~K})$. The results show that, ultimately miniaturized, silicon NEMS can be utilized to realize multi-band, single-chip receiver systems based on NEMS mixer-filter arrays with reduced system complexity and power consumption.

S Online supplementary data available from stacks.iop.org/Nano/23/225501/mmedia
\end{abstract}

(Some figures may appear in colour only in the online journal)

The attraction of NEMS is expressed in their ability to complement or replace key components in information processing systems [1-4]. They are explored as elementary units for memories [5], switches [6] and for quantumstate manipulation [7, 8]. The high quality factor makes mechanical resonators ideal as reference oscillators [9] and selective filters [10], while covering frequencies from $\mathrm{RF}$ to microwave $[11,12]$. An exceptional feature of some electromechanical systems is their ability to combine signal mixing and filtering into one device that becomes equivalent to a full circuit function. Frequency up- and down-conversion is a fundamental concept in modern communication technologies and typically involves at least one filter, mixer, amplifier and local oscillator. These functional elements have been implemented separately and off-chip, which has prevented the ultimate miniaturization of receiver systems. As highly selective signal processing elements, mechanical resonators offer the potential to combine and replace these components by a single device unit, or at least considerably relax their phase noise, dynamic range or linearity requirements [13]. This opens novel avenues for single-chip solutions, which can help to reduce complexity, costs and power consumption. To unlock the full potential of mechanical resonators, however, a number of requirements must be met: their implementation on a massive scale, i.e. in large arrays as opposed to single device demonstrations, their compatibility with existing technologies [14], and a high degree of freedom in the choice of electrical and mechanical design parameters [15].

To date, simultaneous mixing and filtering have been demonstrated in interdigitated silicon micromechanical resonators at very high frequencies $[15,16]$ where, importantly, the mixing originated from the electrostatic nonlinearity of the input transducer (the electrostatic force 
is related to the square of the input voltage, $F_{\mathrm{el}} \sim V^{2}$ ). Basic mixing and demodulation have been demonstrated with carbon based devices using both resonating [17, 18] and non-resonating [19, 20] configurations. However, low quality $(Q-)$ factor, ultra-high vacuum and low temperature operation impose limitations. This is in contrast to top-down, single crystalline silicon which can benefit from a mature technology with the advantage of high process control, high throughput, electronic co-integration and state-of-the-art dimensions approaching those of bottom-up fabrication methods [21].

In this study, we propose a scalable NEMS channelselect receiver, in which mixing and filtering are achieved simultaneously by merging a high- $Q$, nanoelectromechanical resonator with transistor-based signal processing, resulting in an active (or hybrid) resonator. In particular, the device was integrated as a front-end receiver, operating at very high frequencies $(114.4 \mathrm{MHz})$ and near ambient pressure. Figure 1(a) presents the simplified, system-level block diagram of a conventional FM receiver. Its functional units typically involve an RF antenna after which the RF carrier signal is pre-amplified and then down-mixed to an intermediate frequency (IF). The tunable oscillator allows the selection of a respective receiver channel. After IF filtering, the baseband is recovered by the FM discriminator and processed by subsequent baseband electronics. In contrast, figure 1(b) shows the possible front-end architecture taking advantage of the simplicity achievable via the proposed device. Here, the channel selection was readily achieved at $\mathrm{RF}$ frequencies, while the succeeding electronic blocks in the receive path could be condensed into a single device unit on a silicon chip.

Figure 1(c) shows the experimental setup employed in this work. The NEMS receiver was placed in a vacuum probe chamber (Süss PMC150). The gas pressure $\left(\mathrm{N}_{2}\right)$ could be manually adjusted to allow the study of the device as a function of pressure. The tuning fork resonator is represented as a four-terminal device, with input and output (drain and source) terminals and two independent gate electrodes ( $V_{\mathrm{GS} 1}$ and $\left.V_{\mathrm{GS} 2}\right)$. A signal generator (HP8648B) provided the FM signal at the device input. At the output, the recovered baseband signal was routed to a lock-in amplifier, spectrum analyzer, oscilloscope or an audio pre-amplifier. The output signal remained untreated by additional filtering, analyzing the raw performance of the NEMS receiver. For static characterization or dc supply, we used an Agilent Parametric Analyzer 4156C.

The NEMS receiver operation was based on the resonant-body FET. The details of its principle and fabrication have been reported elsewhere [22, 23]. This device is essentially a crystalline silicon resonator, which incorporates a freely suspended transistor. The output of the resonator is simultaneously the drain current signal of the FET, which depends on the mechanical displacement, and peaks at resonance. In such a hybrid resonator, the design of the FET parameters can be set and optimized independently from the mechanical modal shape design. This advantage was demonstrated by integrating n-type, enhancement-mode FETs

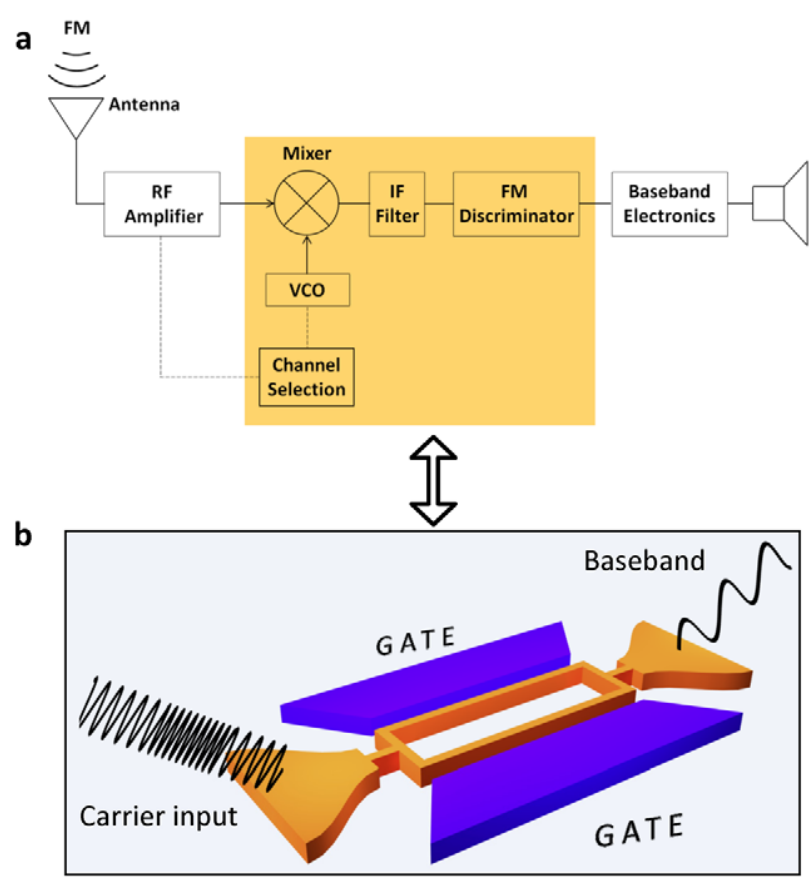

C

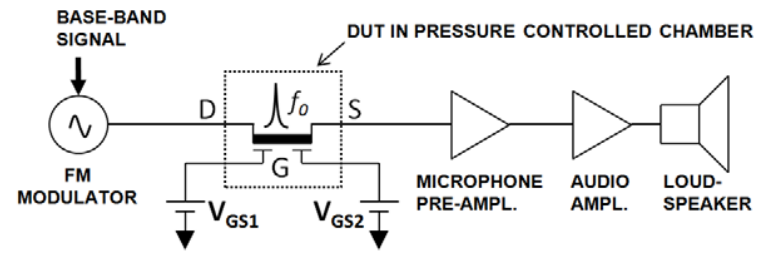

Figure 1. A single active NEMS channel-select RF receiver. (a) Simplified system block diagram representing a conventional homodyne front-end receiver. The components highlighted by the orange area are replaced by the proposed device. (b) Schematic representation of the active tuning fork resonator, consisting of the input and the output terminal (orange), and the two lateral gate electrodes (violet). The NEMS combines channel selection, mixer and demodulator into one functional unit, integrated on-chip. (c) Circuit schematic and signal routing used in the experiment. The NEMS receiver is indicated as a FET with drain (D), source (S) and two independent gate terminals $(G)$.

into the tine(s) of a double-ended tuning fork resonator, which provided a superior performance over their clamped-clamped beam counterparts reported in previous work [22, 23]: due to mutual cancellation of stress waves at the anchors, a higher quality factor could be expected by driving the tuning fork in its anti-phase mode [24]. Further, a two-fold increase in device transconductance and signal output was achieved by operating the two FETs in parallel [25]. A SEM pictures of the device and details of its hybrid properties are shown in figures 2(a)-(e). The tuning fork resonated in-plane and was actuated through lateral, sub-100 nm air-gap capacitors (figure 2(a)). Figures 2(b) and (c) show the fundamental resonance at 114.4 MHz, which corresponded to the symmetric coupled mode and offered a $Q$ of 1100 in high vacuum. On the other hand, the full transistor functionality can be readily seen in figures 2(d) and (e), depicting the transfer characteristic 
a
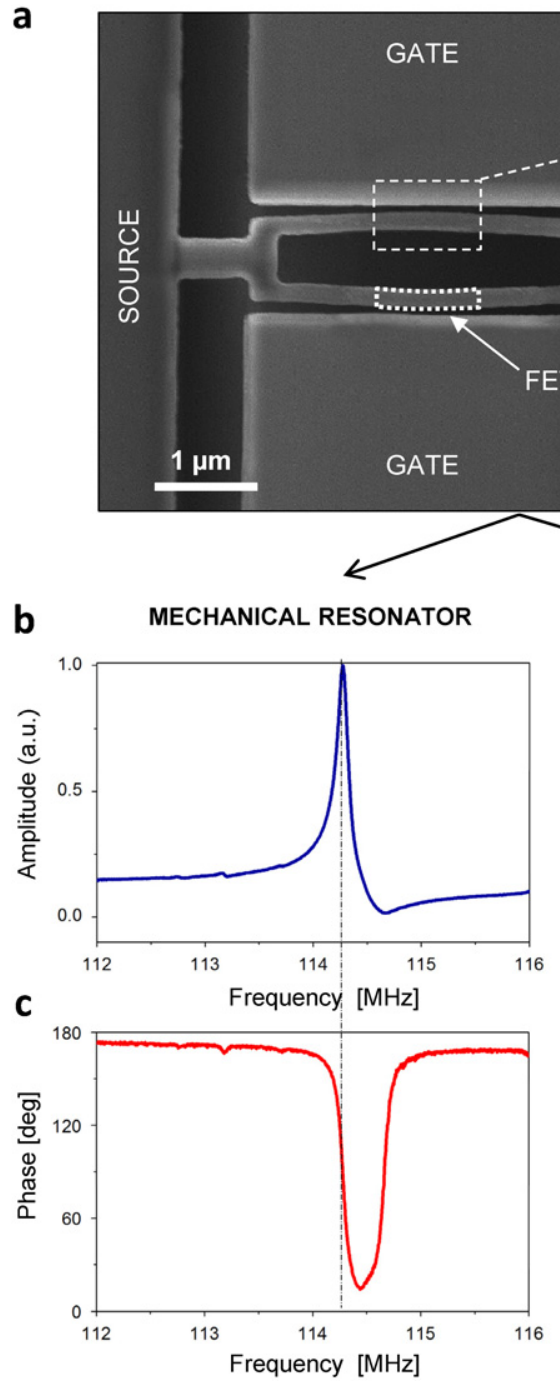
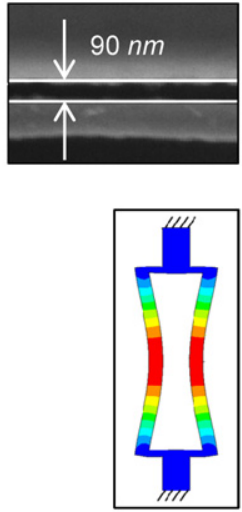

d FIELD EFFECT TRANSISTOR
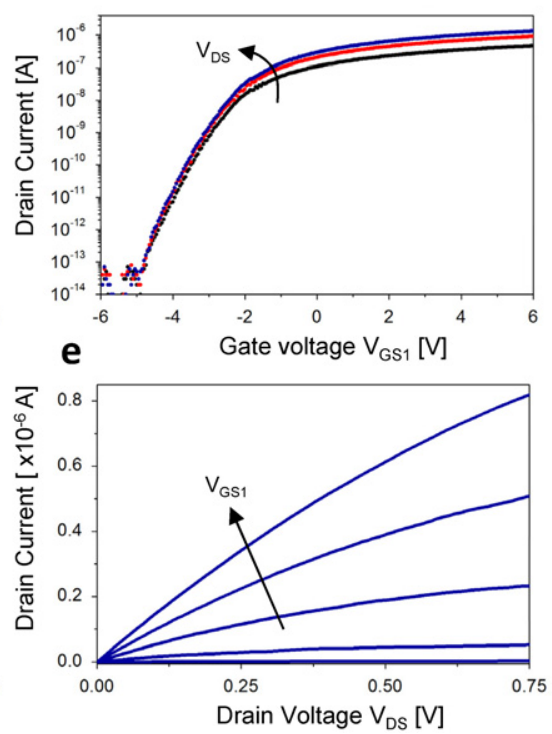

Figure 2. Full transistor functionality incorporated in the NEMS tuning fork resonator. (a) SEM graph of the double-ended tuning fork RF demodulator, with device terminals indicated. The dimensions of the tines are $2.7 \mu \mathrm{m}(l) \times 150 \mathrm{~nm}(w) \times 200 \mathrm{~nm}(t)$. The resonator is actuated laterally on-chip with two independent gate electrodes. The electrostatic gap measures dgap $\sim 90 \mathrm{~nm}$. The FET is integrated in the tuning fork tine. (b), (c) Mechanical properties of the device, showing magnitude and phase responses. The resonance is measured by employing two-source down-mixing as in [22]. The fundamental resonance frequency is found at $f_{0}=114.4 \mathrm{MHz}$ which corresponds to the symmetric coupled mode of the tuning fork. The quality factor is $\sim 1100$ (in high vacuum). The drive conditions are set to $V_{\mathrm{GS} 1 / 2}=11 \mathrm{~V}$, $V_{\mathrm{rf}}=45 \mathrm{mV}$ and $V_{\mathrm{lo}}=63 \mathrm{mV}$. (d), (e) The transfer characteristics $I_{\mathrm{D}}-V_{\mathrm{GS} 1}$ of the transistor for $V_{\mathrm{DS}}=0.1 ; 0.2 ; 0.25 \mathrm{~V}$. The $I_{\mathrm{DS}}-V_{\mathrm{DS}}$ curve of the FET in its linear region, below the knee of the curve, is shown in the inset. The channel conductance varies with the applied gate voltage $V_{\mathrm{GS} 1}=-1 ; 0 ; 1 ; 2 \mathrm{~V}$.

a $I_{\mathrm{DS}}-V_{\mathrm{GS} 1}$ and the output characteristics $I_{\mathrm{DS}}-V_{\mathrm{DS}}$. The well-behaved linear operation of the FET was the underlying principle behind signal mixing in this architecture.

The signal mixing occurred in the NEMS output transducer, i.e. in the transistor, which was used as a resistive mixer $[22,26]$. In this mode, the FET is operated in its linear region, i.e. with no dc drain bias, the FET channel can be represented as a conductance, which is a function of the small-signal drain voltage, the mechanical displacement, and the gate voltage. It can be shown [18] that the term after expansion responsible for frequency demodulation is according to

$$
i_{\text {out }} \propto g_{\mathrm{DS}} v_{\mathrm{in}} \frac{\partial i_{\mathrm{DS}}}{\partial z} z(j \omega)
$$

where $g_{\mathrm{DS}}$ is the differential channel conductance at a given gate voltage $V_{\mathrm{GS}}, i_{\mathrm{DS}}$ is the drain current, $z(j \omega)$ is the instantaneous mechanical displacement, and $\omega$ is the instantaneous frequency. The input FM signal has the general form

$$
v_{\text {in }}(t)=\hat{v}_{\text {in }} \cos [\varphi(t)]
$$

where $\hat{v}_{\text {in }}$ is the carrier amplitude, and the argument is $\varphi(t)=$ $\omega_{\mathrm{c}} t+\frac{\Delta \omega}{\omega_{\mathrm{m}}} \sin \left(\omega_{\mathrm{m}} t\right)$, with $\omega_{\mathrm{c}}$ corresponding to the carrier frequency, $\Delta \omega$ to the frequency deviation, and $\omega_{\mathrm{m}}$ to the modulating signal.

For FM demodulation, i.e. in order to recover the baseband of a FM carrier, a network is required whose output magnitude is proportional to the frequency deviation of the received carrier [27]. Hence, the role of the mechanical 
resonance of the tuning fork in this setup is two-fold: it serves (i) as a carrier filter, with a certain bandwidth $B$, imposed by the natural resonant line width

$$
B \cong \frac{1}{2 \pi} \frac{\omega_{0}}{Q}
$$

and (ii) as a frequency selective network with a transfer function $H(\mathrm{j} \omega)$ that is linked to the mechanical displacement response via

$$
z(j \omega)=\frac{F_{\mathrm{el}}(t)}{m_{\mathrm{eff}}} H(j \omega)
$$

where $F_{\mathrm{el}}(t)$ is the (driving) force on the resonator and $m_{\mathrm{eff}}$ its effective mass. For simplicity, here we approximate the transfer characteristic with a positive and a negative slope $\alpha$ over a certain frequency range around the resonance $\omega_{0} \approx \omega_{\mathrm{c}}$ :

$$
H(j \omega) \cong \pm \mathrm{j} \alpha \omega .
$$

The slope of the filter is dictated by the $Q$-factor. Applying a FM signal of equation (2) to such a filter yields an output according to [27]

$$
v_{\text {out }}(t)=\alpha \hat{v}_{\text {in }}\left[\omega_{\mathrm{c}}+\Delta \omega \cos \left(\omega_{\mathrm{m}} t\right)\right] .
$$

From equations (1) and (6) it can be seen that the resulting output amplitude is a low frequency component which is proportional to the frequency of its input signal, and becomes non-zero only in the presence of a mechanical displacement, which modulates the FET conductance. These device properties allow the retrieval of the baseband signal. When operating the NEMS receiver as described above, the output power of the baseband can be formulated by [26-28]

$$
P_{\text {out }} \propto\left(g_{1} \cdot \beta \cdot \hat{v}_{\text {in }}\right)^{2}
$$

where $\hat{v}_{\text {in }}$ is the input signal amplitude of the FM carrier, $\beta=\Delta f / f_{\mathrm{m}}$ is the modulation index and $g_{1}$ is the fundamental frequency component of the transconductance variation. The variables of equation (7) depend both on transistor and mechanical parameters, which are correlated and given by the device design. We discuss these aspects in the following.

High $Q$-factors are required in oscillator and filter applications. Damping due to gas pressure reduces $Q$ by orders of magnitude. Therefore, MEMS and NEMS devices usually require complex high vacuum packaging which imposes limitations at the wafer scale. Previous work [29] indicates that the damping in the free-molecular flow regime strongly depends on the resonator geometry. In fact, $Q$ of $\mu \mathrm{m}$-sized beams start degrading at lower pressure values compared to those of sub-micrometer scale beams. Therefore, properly scaled resonators have advantages for operation near ambient pressure. Figure 3(a) shows the measured $Q$ as a function of the pressure, which was precisely monitored over orders of magnitude using a Pirani gauge for $p<100$ mbar (Pfeiffer PKR 251) and a piezo gauge for $p>100$ mbar (SMC ZSE30). Here, the drive power was gradually increased to compensate for the damping occurring at an increasing pressure. At pressures below $\sim 1$ mbar, the damping was influenced by various loss mechanisms intrinsic to the resonator and $Q$ became pressure independent. Beyond a

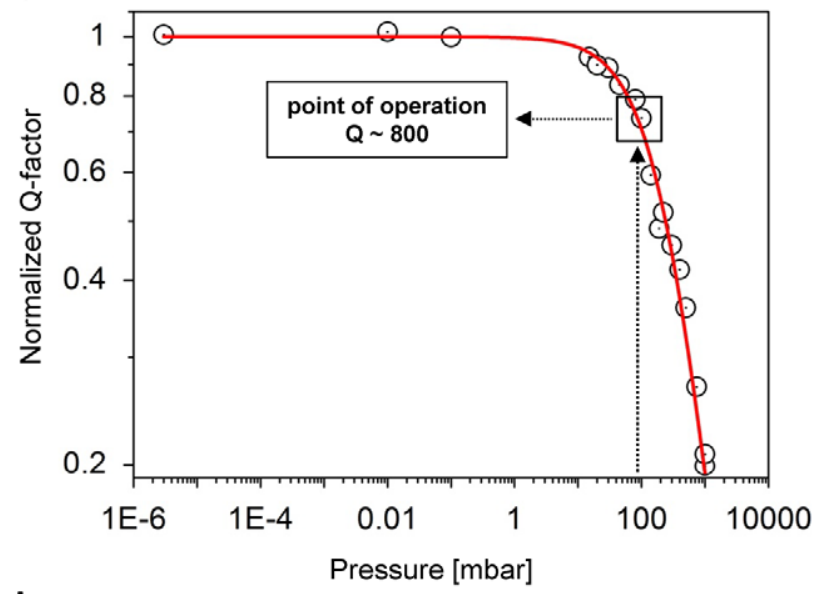

b

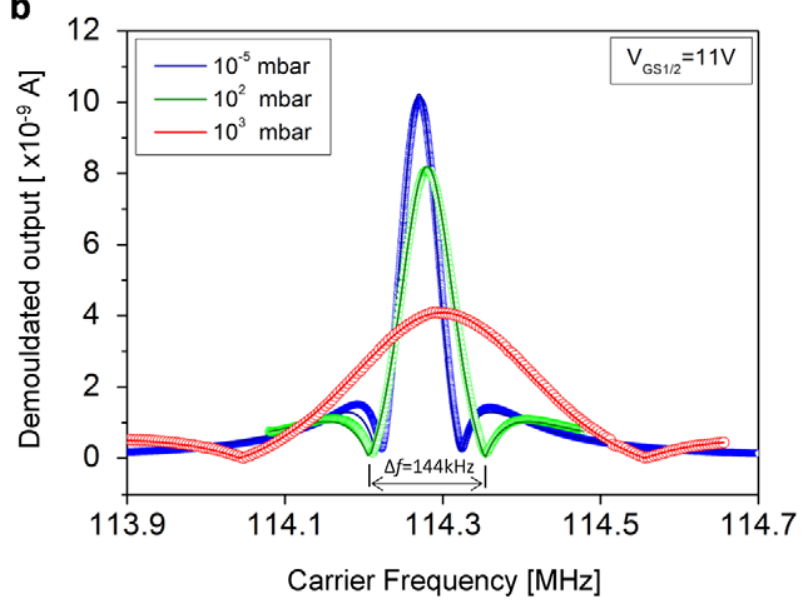

Figure 3. Dependence of NEMS receiver performance on the operating pressure. (a) The $Q$-factor is normalized to its value under high vacuum $\left(Q_{\mathrm{UHV}}=1100\right)$. The extracted $Q$-values agree well with a free-molecular fit (red line). At 100 mbar the Knudsen number is 2.7, based on the resonator thickness. (b) The graph shows the resonance peaks (absolute magnitude) for three different pressures, from high vacuum to ambient pressure, with the typical shape for FM actuation. The drive levels were adapted to the operating conditions: $\Delta f=20 \mathrm{kHz}, V_{\mathrm{ac}}=126 \mathrm{mV}$ for $p=10^{-5}$ mbar; $\Delta f=40 \mathrm{kHz}, V_{\mathrm{ac}}=178 \mathrm{mV}$ for $p=100 \mathrm{mbar}$; and $\Delta f=70 \mathrm{kHz}, V_{\mathrm{ac}}=447 \mathrm{mV}$ for $p=1000$ mbar. The modulation frequency is set constant at $f_{\mathrm{m}}=5 \mathrm{kHz}$.

this point, damping occurred due to momentum exchange of gas molecules impinging on the resonator surface. No deviation of the free-molecular solution from the measured $Q$ values was observed, which indicated that the transition from free-molecular to continuum flow occurred at a pressure corresponding to a Knudsen value above 0.5. This confirmed results that had been obtained in recent experimental work on NEM resonators [30, 31]. The Knudsen number is written as $K_{n}=\lambda_{\mathrm{m}} / T_{b}$, where $\lambda_{\mathrm{m}}$ is the mean free path and $T_{b}$ the transverse dimension normal to the direction of motion subject to fluid interaction. As the mechanical motion is in-plane, $T_{b}$ corresponded here to the thickness of the tuning fork resonator $(\sim 200 \mathrm{~nm})$. For comparison, the mean free path in air under standard conditions is $\sim 65 \mathrm{~nm}$. The pressure of 100 mbar resulted in a resonator $Q$ of $\sim 800$. This operating point was chosen in this work, 
after considering a trade-off between the pressure dependent parameters, i.e. between the bandwidth, signal-to-noise, drive power and power consumption. Pressure values in the low vacuum range remain compliant to industrial standards and would facilitate resonator packaging on the wafer scale, which is of great importance for many technological applications envisioned with NEMS.

The $Q$ of the mechanical resonance directly affected the application space of the NEMS receiver. From equation (6) it can be seen that a larger modulation index $\beta=\Delta f / f_{\mathrm{m}}$ will increase the magnitude (the signal-to-noise) of the recovered signal, albeit at the expense of the bandwidth $B$. For demonstrative purposes, we aimed to comply with communication standards that required the carrier frequency of stations occupying adjacent frequency channels to be separated by $200 \mathrm{kHz}$. For a tentative design, we used Carson's rule to evaluate the bandwidth $B=2\left(\Delta f+f_{\mathrm{m}}\right)$ required to transmit enough power $(>98 \%)$ so that modulation could be recovered without distortion [27]. Figure 4(a) shows the signal recovered by the tuning fork as function of the modulation index, assuming a maximum frequency of modulation $f_{\mathrm{m}}^{\max }=15 \mathrm{kHz}$ for a typical audio signal. At a pressure $p=0.1$ bar the maximum output signal was obtained for a modulation index of $\beta=4$ which required a calculated bandwidth of $B \approx 150 \mathrm{kHz}$. This coincided well with the available resonator line width $f_{0} / Q \approx 144 \mathrm{kHz}$ (figure $3(\mathrm{~b})$ ). Operation of the device at ambient pressure $(p=1$ bar) further improves the signal-to-noise ratio; however, the increasing actuation power and widening of the channel bandwidth could be regarded as factors limiting a practical realization (supporting information available at stacks.iop.org/Nano/23/ $225501 / \mathrm{mmedia})$. As each resonator was equivalent to a single receiver channel, statistical spread in $Q$ will lead to variation in the available bandwidth for each channel. Characterization of 13 tuning fork resonators on dies across the $4^{\prime \prime}$ wafer yielded an average $Q$ of 1074 and a standard deviation of \pm 56 (supporting information available at stacks.iop.org/Nano/23/ $225501 /$ mmedia). Fabrication of top-down, single crystal silicon NEMS with high yield and reproducibility has been demonstrated [21]. Large-scale integration of NEMS remains crucial, e.g. in this application, where arrays of NEMS could be used to cover a broad frequency band in the wireless spectrum, while the gate voltage could be exploited for fine tuning of the resonance frequency (supporting information available at stacks.iop.org/Nano/23/225501/mmedia).

Figure 4(b) shows the output spectrum of a pure sine wave, demodulated by the NEMS receiver operating at 100 mbar. The carrier signal with an input power $P_{\text {in }}=$ $-8 \mathrm{dBm}$ was set equal to the mechanical resonance $f_{0}=$ 114.399 MHz. This frequency point of operation allowed us to exploit the full bandwidth of the mechanical resonance. The NEMS receiver had a measured conversion loss of $\sim 90 \mathrm{~dB}$, which had been determined with $50 \Omega$ measurement instrumentation. Several factors could be attributed to the relatively large conversion loss of the prototyped device. For the FET used in the resistive mode of operation $\left(V_{\mathrm{DS}}=0 \mathrm{~V}\right)$, there will be an optimum gate bias for a given input power that would ideally occur somewhere below the transistor a
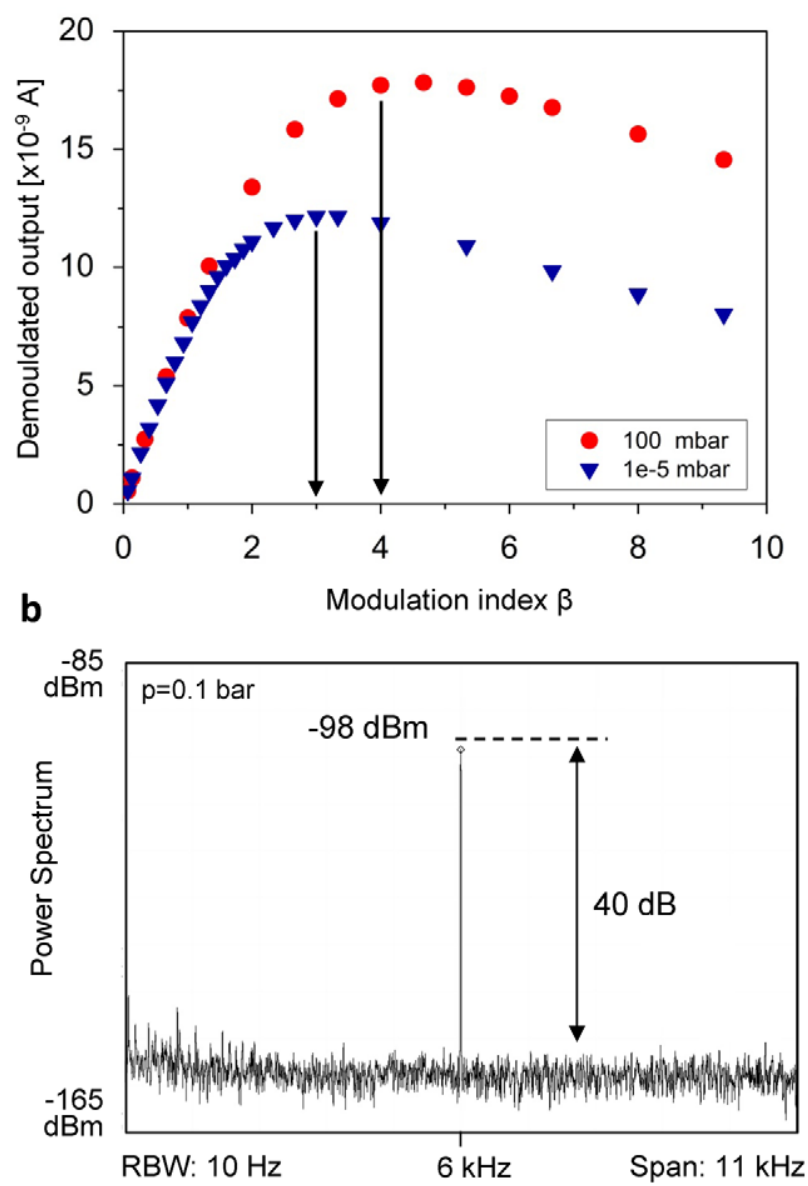

Figure 4. Near ambient pressure operation of the NEMS tuning fork receiver. (a) The peak (FM) demodulated output versus the modulation index $\beta=\Delta f / f_{\mathrm{m}}$ in high vacuum (blue) and at $100 \mathrm{mbar}$ (red), assuming a maximum modulation frequency of $f_{\mathrm{m}, \max }=15 \mathrm{kHz}$. The output saturates with the signal bandwidth approaching the natural line width of the resonator. The bias is $V_{\mathrm{GS}}=11 \mathrm{~V}, V_{\mathrm{ac}}=90 \mathrm{mV}$ at $10^{-5} \mathrm{mbar}$, and $V_{\mathrm{ac}}=178 \mathrm{mV}$ at $p=100$ mbar, respectively. (b) Output spectrum of the NEMS receiver for a sinusoidal input at 100 mbar operating pressure $\left(f_{\mathrm{m}}=6 \mathrm{kHz}, \Delta f=40 \mathrm{kHz}\right)$. The carrier to noise ratio is $\sim 51 \mathrm{~dB}$ at $1 \mathrm{~Hz}$ resolution, the carrier input power $P_{\text {in }}=-8 \mathrm{dBm}$.

threshold voltage [26] in order to maximize the component $g_{1}$ of equation (6) (supporting information available at stacks.iop.org/Nano/23/225501/mmedia). Therefore, for an optimized device design, a threshold voltage (here $V_{\text {th }} \sim$ $-2 \mathrm{~V})$ coinciding with the dc voltage $\left(V_{\mathrm{GS}}=+11 \mathrm{~V}\right)$ required to efficiently actuate the NEMS, would be desirable. Further, device input and output should be impedance matched to the source and load, respectively, at the frequencies of interest. In this way, the signal-to-noise in this device could be improved from 9 to $46 \mathrm{~dB}$, taking into account a required bandwidth of $15 \mathrm{kHz}$ for audio signals. Figure 5(a) shows the corresponding measurements in the time domain. The recovered baseband signal was of the order of $5 \mathrm{mV}$ peak-peak across $1 \mathrm{M} \Omega$. It showed little distortion and very symmetric half-waves. We emphasize that all signal operations up to this point were performed with zero dc power consumption and an ac power consumption of a few hundred microwatts. At an 


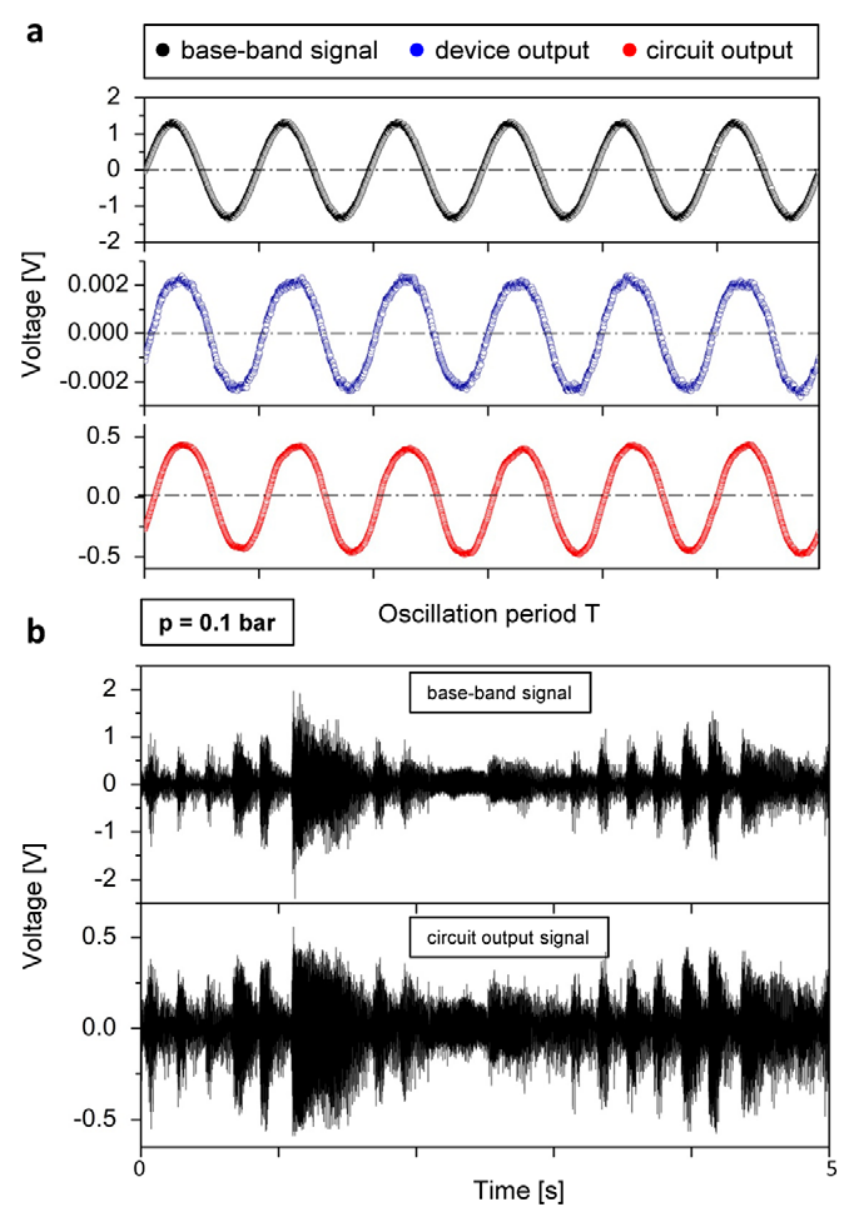

Figure 5. Output waveform recovered by the NEMS receiver at near ambient pressure. (a) Time domain measurement for a sinusoidal baseband signal at an operating pressure $p=100 \mathrm{mbar}$ $\left(f_{\mathrm{m}}=3 \mathrm{kHz}, \Delta f=35 \mathrm{kHz}, P_{\text {in }}=-6 \mathrm{dBm}\right)$. The output signals are measured on an oscilloscope with $1 \mathrm{M} \Omega$ input impedance. The device output waveform is averaged by a factor of 16 . The signal amplitude is symmetrically distributed around the zero-volt value (dash-dotted line). Detuning from the resonance frequency leads to a shift of the waveform upwards or downwards, respectively. (b) Input and output waveform of an audible audio file (Beethoven's sonata Pathétique, Allegro), unfiltered and directly amplified from the NEMS output terminal.

operating pressure of 0.1 bar, the best results were obtained for a frequency deviation of $\Delta f \approx 35 \mathrm{kHz}$ (or $\beta \approx 2.3$ ) which corresponded to the upper limit of linear operation (see figure 4(a)). For higher modulation indices, higher harmonic current components caused distortion in the output signal as described recently in the literature [18]. We used a commercial microphone pre-amplifier (supporting information available at stacks.iop.org/Nano/23/225501/mmedia) to attain a $400 \mathrm{mV}$ peak-peak output signal and have recorded $5 \mathrm{~s}$ of Beethoven's sonata Pathétique (the Allegro). Without any further filtering, the waveform was recovered with good fidelity as shown in figure 5(b) (the .wav-file is found in supporting information available at stacks.iop.org/Nano/23/225501/mmedia).

In conclusion, we have demonstrated a novel radio receiver architecture based on a nanoelectromechanical system which combines the gain in a transistor with the large $Q$ of a tuning fork mechanical resonator. Both elements are synthesized in a single device, enabling on-chip functions previously inaccessible in silicon. We demonstrate electromechanical demodulation of FM signals at VHF $(>100 \mathrm{MHz}$ ) with the single active NEM resonator, which represents one receiver channel and can be arranged in large arrays to cover whole frequency bands. The operation at near ambient pressure maintains a high $Q=800$ and a low level of power consumption in the sub-milliwatt range. As such, this work represents a major step towards practical and useful realization of NEMS-based systems, which can benefit from more functionality, high levels of integration, low power consumption and reduced complexity at system level.

\section{Acknowledgments}

This work was supported by the European Framework Project FP7 NEMSIC 'Hybrid Nano-Electro-Mechanical Systems for Sensing and Power Management Applications'. We thank A Lovera, D Grogg and the staff of the EPFL Centre for MicroNanotechnology (CMI) for assistance in device fabrication.

\section{References}

[1] Nguyen Clark T C 2005 MEMS technology for timing and frequency control Proc. IEEE Trans. Ultrason. Ferroelec. Freq. Control 54 251-70

[2] Mahboob I, Flurin E, Nishiguchi K, Fujiwara A and Yamaguchi H 2011 Interconnect-free parallel logic circuits in a single mechanical resonator Nature Commun. 2198

[3] Lee S W, Park S J, Campbell E E B and Park Y W 2011 A fast and low-power microelectromechanical system-based non-volatile memory device Nature Commun. 2220

[4] Feng X L, White C J, Hajimiri A and Roukes M L 2008 A self-sustaining ultrahigh-frequency nanoelectromechanical oscillator Nature Nanotechnol. 3 342-6

[5] Mahboob I and Yamaguchi H 2008 Bit storage and bit flip operations in an electromechanical oscillator Nature Nanotechnol. 3 275-9

[6] Abele N et al 2005 Suspended-gate MOSFET: bringing new MEMS functionality into solid-state MOS transistor Tech. Dig. IEDM 1609384

[7] Massel F et al 2011 Microwave amplification with nanomechanical resonators Nature 480 351-4

[8] Verhagen E, Deleglise S, Weis S, Schliesser A and Kippenberg T J 2012 Quantum-coherent coupling of a mechanical oscillator to an optical cavity mode Nature 482 63-7

[9] Otis B P and Rabaey J M 2003 A 300-uW 1.9-GHz CMOS oscillator utilizing micromachined resonators IEEE J. Solid-State Circ. 38 1271-4

[10] Bannon F D, Clark J R and Nguyen C T C 2000 High- $Q$ HF microelectromechanical filters IEEE J. Solid-State Circ. 35 512-26

[11] van Beek J T M et al 2007 Scalable $1.1 \mathrm{GHz}$ fundamental mode piezo-resistive silicon MEMS resonator Tech. Dig. IEDM 4418960

[12] Weinstein D and Bhave S A 2010 The resonant body transistor Nano Lett. 10 1234-7

[13] Nguyen C T C 1999 Frequency-selective MEMS for miniaturized low-power communication devices IEEE Trans. Microw. Theory 47 1486-503

[14] Nguyen C T C and Howe R T 1999 An integrated CMOS micromechanical resonator high- $Q$ oscillator IEEE J. Solid-State Circ. 34 440-55 
[15] Wong A C and Nguyen C T C 2004 Micromechanical mixer-filters ('mixlers') J. Microelectromech. Syst. $13100-12$

[16] Ngyuen C T, Gutnik V and Howe R T 1998 Mixing, modulation and demodulation with electromechanical resonators US Patent Specification 5,839,062

[17] Jensen K, Weldon J, Garcia H and Zettl A 2007 Nanotube radio Nano Lett. 7 3508-11

[18] Gouttenoire V et al 2010 Digital and FM demodulation of a doubly clamped single-walled carbon-nanotube oscillator: towards a nanotube cell phone Small 6 1060-5

[19] Rutherglen C and Burke P 2007 Carbon nanotube radio Nano Lett. 7 3296-9

[20] Rodriguez-Morales F et al 2006 Direct and heterodyne detection of microwaves in a metallic single wall carbon nanotube Appl. Phys. Lett. 89083502

[21] Mile E et al 2010 In-plane nanoelectromechanical resonators based on silicon nanowire piezoresistive detection Nanotechnology 21165504

[22] Bartsch S T, Lovera A, Grogg D and Ionescu A M 2012 Nanomechanical silicon resonators with intrinsic tunable gain and sub-nw power consumption ACS Nano 6 256-64
[23] Grogg D and Ionescu A M 2011 The vibrating body transistor IEEE Trans. Electron Devices 58 2113-21

[24] Yan J and Seshia A A 2009 Thermoelastic dissipation in mems/nems flexural mode resonators $J$. Nanosci. Nanotechnol. 9 1011-4

[25] Bartsch S T, Lovera A, Grogg D and Ionescu A M 2011 Very high frequency double-ended tuning fork FIN-FET resonator IEEE Int. Conf. Solid-State Sensors Actuators, Microsyst. 938-41

[26] Maas S A 1987 A GaAs-MESFET mixer with very low intermodulation IEEE Trans. Microw. Theory 35 425-9

[27] Taub H and Schilling D L 1986 Principles of Communication Systems 2nd edn (New York: McGraw-Hill)

[28] Peng S T 1997 A simplified method to predict the conversion loss of FET resistive mixers IEEE Mtt-S pp 857-60

[29] Bhiladvala R B and Wang Z J 2004 Effect of fluids on the $Q$ factor and resonance frequency of oscillating micrometer and nanometer scale beams Phys. Rev. E 69036307

[30] Li M W et al 2008 Bottom-up assembly of large-area nanowire resonator arrays Nature Nanotechnol. 3 88-92

[31] Li M, Tang H X and Roukes M L 2007 Ultra-sensitive nems-based cantilevers for sensing, scanned probe and very high-frequency applications Nature Nanotechnol. 2 114-20 